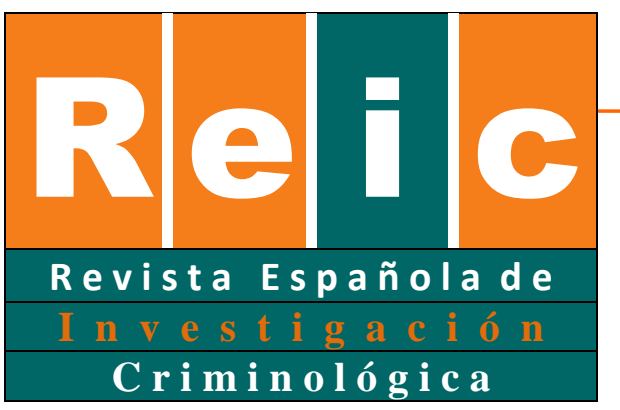

Díaz-Fernández \& del Real Castrillo

\title{
La investigación con temas sensibles en los Trabajos Fin de Grado en Criminología
}

\section{Investigating sensitive topics in the End-of-Degree Assignment (EDA) on Criminology}

\author{
Antonio M. Díaz-Fernández ${ }^{1}$ iD , y Cristina del Real Castrillo (D) \\ Universidad de Cádiz
}

\section{RESUMEN}

Una parte importante de los Trabajos de Fin de Grado (TFG) en Criminología se realizan sobre temas considerados sensibles. Este artículo analiza cómo las primeras promociones de egresados los han llevado a cabo considerando las importantes implicaciones en la seguridad de los participantes y estudiantes, la adecuada gestión de sus sentimientos y emociones, y el riesgo de que un mal desarrollo del trabajo queme el campo para ulteriores promociones. Para el análisis, se analizaron las experiencias de 83 graduados y graduadas en Criminología (43\% del universo estimado de los estudiantes de Criminología que habían realizado sus TFG en universidades españolas) en torno a las fases del proceso de investigación desde el inicio del trabajo de campo hasta su finalización. Las respuestas se analizaron conforme a las variables: i) percepción de riesgo, ii) valoración del método de investigación, iii) empleo de medidas de seguridad, iv) relación con el grupo y, v) emociones y sentimientos. La investigación generó una tipología de estudiantes cuyo análisis en profundidad se abordará en futuras investigaciones.

Palabras clave: Criminología, seguridad, emociones, temas sensibles, metodología, Trabajo de Fin de Grado (TFG).

\footnotetext{
${ }^{1}$ La correspondencia debe dirigirse a: Antonio M. Díaz-Fernández. Facultad de Derecho, Universidad de Cádiz, Avda. de la Universidad s/n, 11407 Jerez de la Frontera, Cádiz. antonio.diazfernandez@uca.es 


\begin{abstract}
An important part of the End-of-Degree Assignment (EDA) in Criminology is done on sensitive topics. After the first promotions of graduates, this article analyzes how the Criminology students conducted their EDA due to their important implications in the safety of the participants and the investigators, the bad management when leaving the fieldwork that burns it for the further promotions, and an inadequate management of the feelings and emotions of the students. For the analysis, the experiences of 83 graduates in Criminology (43\% of the universe of the Criminology students who conducted their EDA in Spanish universities) were analyzed, through the different phases of the research process from the access to the leaving of the field. The answers were analysed according to: i) the perception of risk, ii) the evaluation of the research method, iii) the use of safety measures, iv) the relationship with the group and v) the emotions and feelings. The answers work out a typology of students whose in-depth analyzes are addressed in future research.
\end{abstract}

Keywords: Criminology, security, emotions, sensitive topics, methodology, End-of-Degree Assignment (EDA).

\title{
1. Introducción
}

Cualquier investigación que se produzca sobre seres humanos es "sensible" en sí misma, ya que todos los seres humanos tenemos una intimidad, unos valores, unos miedos, unas opiniones o unas creencias que proteger del conocimiento externo. De hecho, como decía Barnes (1979, pp.22), “toda investigación social encierra la posibilidad de destruir la privacidad y la autonomía del individuo, de proporcionar más poder a aquellos que ya lo ostentan, de crear la infraestructura para un Estado opresor invencible”. En la Criminología esta característica se enfatiza debido a su propio objeto de estudio, esto es, delito, delincuente, víctima y control social. Por tanto, los TFG que se realizan en el Grado en Criminología ${ }^{2}$-a diferencia de otros Grados universitarios- tienen altas posibilidades de albergar temas sensibles siempre y cuando se elaboren mediante el desarrollo de un trabajo de campo y, por tanto, se interactúe con individuos y colectivos.

A pesar de que la literatura científica ha mostrado en extenso que el trabajo con estos temas puede comportar riesgos físicos, psicológicos y legales, cada curso académico,

\footnotetext{
${ }^{2}$ A efectos de este artículo, las referencias al Grado en Criminología engloban a todas las variantes existentes en las Universidades españolas tales como Criminología y Seguridad o Criminología y Políticas Públicas de Seguridad.
}

Revista Española de Investigación Criminológica

Artículo 2, Número 16 (2018)

https://doi.org/10.46381/reic.v16i0.147

WWW.criminologia.net

ISSN: 1696-9219 
estudiantes sin entrenamiento específico se apresuran en la elaboración de estos trabajos sin una reflexión previa sobre el hecho de que van a trabajar con seres humanos. Lo realizan sin reparar en si aquella información podría haber sido obtenida de forma menos intrusiva o traumática para los participantes y, en ocasiones, desapareciendo de manera súbita del campo una vez obtenido lo buscado, dificultando así, con cada promoción que pasa, que otros estudiantes puedan acudir al mismo campo al quedar parcialmente quemado. Junto a esto, el trabajo con temas sensibles es un recurso pedagógico que no ha sido explorado sino en exiguo. Esto es llamativo considerando que no gestionar las posibles emociones experimentadas puede tener un impacto en estos jóvenes estudiantes que se han enfrentado, entre otros, a delincuentes, prostitutas, mujeres maltratadas o inmigrantes a punto de ser expulsados del país, afectando en potencia también a la fiabilidad y validez de la información obtenida.

Todo esto supone que los estudiantes deban plantearse desde el mismo inicio del trabajo de campo cuál será el equilibrio entre el beneficio para la Ciencia y la protección de los participantes. Por otro lado, habrán de reflexionar sobre si todo ese efluvio de emociones, que necesariamente se va a producir entre el aula y la calle y entre la calle y el aula, son canalizadas adecuadamente de forma que se pueda generar un aprendizaje en y a través de las emociones como parte de los contenidos del Grado.

La necesidad de comprender el cómo se elaboran los TFG no es un aspecto menor considerando la carga lectiva que representan y su lugar culminante en los Grados universitarios. El Real Decreto 1393/2007, de 29 de octubre, establecía en el artículo 12.3 que las enseñanzas conducentes a la obtención de un título de grado "concluirán con la elaboración y defensa de un trabajo de fin de Grado", que: i) debe tener una carga de al menos 6 créditos y un máximo del $12.5 \%$ del total de créditos del curso, ii) debe ser realizado en la fase final del plan de estudios, y iii) debe estar orientado a la evaluación de competencias asociadas al título. La aplicación práctica por parte de las Universidades ha sido heterogénea, tanto en el número de créditos que le han asignado como en las modalidades de trabajos. Así, por ejemplo, mientras en la Universidad de Sevilla el TFG tiene una carga lectiva de 6 créditos, en la Universidad del País Vasco es la nada desdeñable de 18 créditos. 
En definitiva, tras las primeras promociones de egresados, los autores se plantearon analizar este novedoso este instrumento pedagógico como es el TFG en nuestro país. A la novedad se unía la particularidad de que la temática de muchos de ellos puede ser considerada sensible. El objetivo era, por tanto, proponer, por una parte, mejoras en la tutorización y, por otra, explorar el papel que juegan las emociones en los TFG con la pretensión de que sirvan como complemento al desarrollo de las competencias y habilidades que los Grados universitarios deben procurar para estudiantes que se enfrentan a colectivos que pueden estar muy lejanos de su realidad social y cultural.

Esta interacción con los participantes supone un reto para el habitual aprendizaje unidireccional de los estudiantes universitarios. La necesidad de que los participantes comprendan tanto las garantías y protección que merecen como, por otra parte, qué tipo de competencias se está buscando desarrollar en el joven universitario pasa por explicarles que, más allá del aprendizaje teórico, se está trabajando en la dimensión personal. De esta forma, se podrá favorecer un desarrollo de competencias en los estudiantes que redundará en la generación de profesionales más empáticos y comprometidos con diferentes realidades sociales, en muchas ocasiones, alejadas de la suya.

En 1988, Sieber y Stanley establecieron que una investigación sensible era aquélla en la cual existían unas consecuencias o implicaciones potenciales tanto para los participantes directos como para aquellos individuos representados por el estudio. Pero quienes se han dedicado al tema con mayor intensidad y autoridad han sido Renzetti y Lee que defienden que un tema sensible es aquél que "potencialmente puede suponer para aquellos implicados una amenaza sustancial, la emergencia de la cual puede ser problemática para el investigador o para los investigados por la recopilación, posesión y la difusión de datos” (1993, pp.5); es decir, amplían el rango de los potenciales sufridores al incluir a los investigadores. Debido a los difusos contornos de este término, Renzetti y Lee (1993) reconocen su preferencia más que por hacer una lista exhaustiva de temas sensibles, por analizar bajo qué condiciones surge la "sensibilidad". Para ello, facilitaron cuatro grandes entornos: i) aquél en el que la investigación invade la esfera privada o escarba dentro de alguna experiencia personal profunda, ii) cuando se hace referencia a la desviación o control social, iii) cuando se afecta a los intereses de personas poderosas o el ejercicio de la coerción y la dominación y, iv)

\section{Revista Española de Investigación Criminológica}

Artículo 2, Número 16 (2018)

www.criminologia.net

ISSN: 1696-9219 
cuando se lidia con cosas sagradas para los estudiados y que estos no quieren que sean profanadas. Aquellos temas que se engloben dentro de estos cuatro entornos serán considerados como sensibles para los investigadores a los efectos de seleccionar los TFG objeto de análisis.

Aunque toda la investigación en temas sensibles puede tener consecuencias, algunos grupos de personas deberán lidiar con ellas más que otros. A estos grupos de personas o poblaciones se los conoce como vulnerables, término que engloba a aquellas personas que tienen la capacidad de autonomía disminuida debido a factores físicos, psicológicos, o desigualdades sociales (Silva, 1995), y cuya manifestación es una menor capacidad para tomar elecciones personales sobre su vida o mantener su independencia. Es por ello que estas personas necesitan, a menudo, de una especial protección que garantice sus derechos y su bienestar, a lo que se añade que muchos han sido víctimas de algún daño o están en riesgo de sufrirlo (Moore \& Miller, 1999). Este término también puede hacer referencia a grupos sociales que corren riesgo de sufrir algún perjuicio en su salud (Flaskerud \& Winslow, 1998), o de padecer discriminación, intolerancia y estigmatización (Nyamathi, 1998).

Existe una extensa producción científica sobre temas sensibles, no obstante, los autores de este artículo conocen limitadas investigaciones que tengan como objeto específico a estudiantes universitarios ya que, en su mayor parte, se centran en investigadores doctorales o senior. Por ejemplo, Macklin (1980), Mosher (1988) y Wolf (2009) que sí analizaron los problemas surgidos con los trabajos realizados por estudiantes, solo hacen mención a la falta de inclusión del riesgo psicológico en los mismos, algo que ya habían apuntado Mead (1972) y Howell (1990). El estudio de Dickson-Swift, James, Kippen y Liamputtong (2007) recoge entrevistas realizadas a 30 investigadores que trabajan con temas sensibles y riesgo psicológico, si bien son mínimas las referencias a otro tipo de riesgos en trabajos de investigación elaborados por universitarios.

Low y Organista (2000) creen que, para los estudiantes, la Universidad es un buen lugar para aprender y debatir sobre temas sensibles. En su experiencia, aquellos estudiantes que los trabajan en clase los encuentran menos difíciles; probablemente debido a que alcanzan una mayor confianza y conocimiento, al tiempo que adquieren habilidades para trabajar de forma cómoda con ellos, reflexión que coincide con la más reciente de Lowe 
(2015). Nos encontraríamos, por tanto, ante el reto que lanzaba O’Byrne (2014) de "transformar el aula en un espacio de sentimientos", en nuestra opinión, un magnífico complemento a la docencia teórica.

La falta de preparación a los estudiantes tiene parte de su origen en los propios docentes/investigadores y su (nuestra) gran obsesión por mostrar las investigaciones como puras y con datos recopilados de manera sistemática cuando, de hecho, este trabajo de campo se ha producido en entornos de confusión, amenazas, peligro e impredecibilidad (Nilan, 2002). Para Bryman (1988), esto provoca que los futuros investigadores crezcan con manuales que presentan las investigaciones como un proceso estricto e higiénico, por lo que acaban creyendo que los problemas provienen más de su incapacidad que de la dificultad del objeto al que se aproximan. Además, tampoco las emociones de los investigadores suelen ser parte del cuerpo de los artículos (Dickson-Swift et al., 2009) porque, como Kleinman (1991) argumenta, si lo hubieran hecho hubieran pasado como menos fiables para sus colegas cuantitativos; en conclusión, el estudiante da sus primeros pasos con modelos de "perfección" y "frialdad emocional".

Las fases del acceso, trabajo y salida del campo son las mismas que para cualquier otra investigación. La diferencia estriba -junto a la, a veces, complejísima tarea de encontrar a individuos y colectivos que están ocultos, para lo que hay que recurrir a gatekeepers específicos- en la necesidad de desarrollar los mecanismos de protección suficientes para garantizar la seguridad de los participantes y del propio investigador. La necesidad de tomar decisiones de manera constante en un entorno sociocultural que no suele ser el habitual del investigador conlleva un intenso trabajo preparatorio.

Considerando tanto las fases de la investigación como aquellos elementos donde mayores peculiaridades se producen en los estudios con temas sensibles, los resultados fueron analizados en relación con las siguientes variables y que serán analizadas en detalle en el apartado 3:

i) La percepción de riesgo se ha analizado como un constructo formado por dos entidades: la percepción, entendida como la elaboración e interpretación de las sensaciones adquiridas por los sentidos, y el riesgo, definido como la probabilidad de que 
el sujeto de investigación sufra algún daño físico, psicológico o moral como consecuencia inmediata o tardía de los métodos de investigación utilizados.

ii) La valoración del método de investigación, en la que se han incluido aquellos ítems en los cuales el estudiante daba su opinión sobre el método de investigación que había utilizado. En concreto, se ha tenido en cuenta la visión global del método de investigación y el análisis sobre el lugar donde se desarrolló el trabajo de campo.

iii) El empleo de medidas de seguridad se ha entendido como el conjunto de todas aquellas medidas utilizadas para evitar o reducir los efectos de los riesgos tanto para los participantes como para el propio estudiante, que se encontrará en entornos desconocidos y alejados de su realidad sociocultural.

iv) La relación con el grupo se ha construido con las respuestas sobre la influencia de la apariencia externa de los estudiantes en la actitud de los participantes, así como la actitud de los propios estudiantes hacia estos.

v) Las emociones y sentimientos en la investigación, experimentados por el estudiante durante el trabajo de campo y en su salida del mismo.

\section{Método}

\subsection{Muestra}

Para calcular el universo para este estudio se solicitó información a las Universidades españolas que imparten el Grado en Criminología y que contaran al menos con una promoción de egresados. De las respuestas obtenidas y de los datos de sus páginas webs se extrajo que este Grado lo han finalizado aproximadamente un 45\% de los estudiantes que se matricularon desde su implantación hasta el curso 2015-16; es decir, 2371 alumnos de 5270 y que son, por consiguiente, quienes habrían elaborado su TFG. Para establecer cuántos de estos TFG implicaban la realización de trabajo de campo -y por tanto ser susceptible de llevarse a cabo sobre temas sensibles- realizamos un análisis de todos los TFG de este Grado de las Universidades de Cádiz, Sevilla y Pompeu Fabra. Conforme a este análisis, extrajimos que en torno a un 10\% de los trabajos implican labor de campo; es decir, 237, y de ellos, 
comprobamos que un $82 \%$ versaba sobre temas que son considerados sensibles, dando de esta forma lugar a un universo aproximado para nuestro estudio de 194 TFG.

Se recibieron 111 respuestas, si bien la muestra final quedó compuesta por las de 83 egresados en Criminología que habían realizado sus TFG en algún tema sensible; este número supone un $43 \%$ de los 194 que habíamos establecido como universo. La criba se produjo seleccionando aquellos que habían llevado a cabo trabajo de campo rechazando, por ejemplo, aquellos TFG que eran de mera revisión bibliográfica. La muestra final quedaba compuesta por 26 hombres $(31.3 \%)$ y 57 mujeres $(68.7 \%)$ con una representación de casi todas las Universidades españolas con Grado en Criminología. El 81.3\% de los trabajos de la muestra se habían confeccionado en los cursos 2014/15 (33.7\%) y 2015/16 (49.4\%).

La temática desagregada de los TFG objeto de la muestra queda recogida en la Tabla 1. Estos temas se corresponden, en opinión de los autores, con aquellos que con mayor frecuencia se trabajan en los seminarios y prácticas del Grado en Criminología y, en no pocas ocasiones, las principales áreas de especialización de los docentes que están impartiendo estos Grados en las diferentes Universidades españolas. Así, prisiones (17\%), menores (13.4\%), violencia de género (12.5\%) y marginación social/urbana (10.7\%) engloban el $53.6 \%$ de todos los TFG de la muestra.

\section{Tabla 1.}

Muestra de temas sensibles en los TFG

\begin{tabular}{|l|c|}
\hline Prisiones & 17 \\
\hline Menores & 13.4 \\
\hline Violencia de género & 12.5 \\
\hline Marginación social/urbana & 10.7 \\
\hline Prostitución & 5.4 \\
\hline Crimen organizado & 4.5 \\
\hline LGTB & 4.5 \\
\hline Drogas & 3.6 \\
\hline Política & 2.7 \\
\hline Extranjería & 2.7 \\
\hline Percepción de seguridad & 2.7 \\
\hline Personas mayores & 2.7 \\
\hline Aborto & 1.8 \\
\hline Corrupción & 1.8 \\
\hline Enfermedades mentales & 1.8 \\
\hline
\end{tabular}




\begin{tabular}{|l|c|}
\hline Pobreza & 1.8 \\
\hline Terrorismo & 1.8 \\
\hline Pornografía & 0.9 \\
\hline Eutanasia & 0.9 \\
\hline Transexualidad & 0.9 \\
\hline Robos & 0.9 \\
\hline Jurado popular & 0.9 \\
\hline Movimientos sociales & 0.9 \\
\hline Racismo & 0.9 \\
\hline Violencia en el trabajo & 0.9 \\
\hline Víctimas & 0.9 \\
\hline Violencia en el Deporte & 0.9 \\
\hline Total & $\mathbf{1 0 0}$ \\
\hline
\end{tabular}

\subsection{Instrumento}

Para recopilar la información se elaboró un cuestionario en Google Docs ${ }^{3}$ que, si bien frente a otras opciones obligaba al participante a tener una cuenta en Google, impedía al mismo tiempo que una persona rellenase más de una encuesta. Los 76 ítems del cuestionario eran tanto preguntas abiertas $(\mathrm{N}=15)$ como preguntas cerradas $(\mathrm{N}=61)$, aunque una de ellas ("técnica de investigación empleada") hacía de filtro para redirigir al estudiante a la sección que le correspondía, por lo que el número final de preguntas era siempre menor.

Estructuramos el cuestionario en torno a las fases de la investigación que el alumno ha de seguir y, por tanto, en los momentos en los cuales los problemas de trabajar con temas sensibles deben aparecer y las potenciales soluciones adoptarse. Esta estructura engloba todos los elementos que han de abordar los investigadores sociales que se enfrentan con los temas sensibles y que, como indica Brewer (1993), son de carácter metodológico, técnico, ético, de contexto social y de seguridad personal. El esquema final del cuestionario quedaba por tanto compuesto por los siguientes ítems: i) datos de identificación personal (8 ítems), ii) elección del tema (7 ítems), iii) acceso al campo (11 ítems), iv) trabajo de campo (3 ítems) y, dentro de éste, la pregunta filtro dirigía hacia: a) entrevistas en profundidad (10 ítems) o

\footnotetext{
${ }^{3}$ El cuestionario está disponible en el siguiente enlace: https://goo.gl/forms/vBd7WpR8Iz4Gp8Is2
} 
b) observación participante ( 6 ítems), v) relaciones con los participantes ( 9 ítems), vi) protección (14 ítems), y vii) salida del campo (8 ítems).

\subsection{Procedimiento}

El trabajo de campo se realizó entre marzo y mayo de 2016. El enlace al cuestionario se envió a través de un correo individualizado a los coordinadores de los Grados en Criminología de toda España con la petición de que lo hicieran llegar a sus egresados. Se difundió también a través de las cuentas de Twitter y Facebook de las Asociaciones de Estudiantes en Criminología. También se realizaron invitaciones individuales a través de las redes sociales a autores de TFG que estaban disponibles a través de repositorios y cuyo título nos indicaban que podían encajar en el universo.

El análisis de los datos se desarrolló con el paquete estadístico SPSS. Se realizó un análisis descriptivo de la encuesta y se elaboraron tablas de contingencia para comprobar cómo se relacionaban las variables entre sí. Las respuestas cualitativas pasaron por un proceso de categorización en el que fueron transformadas en datos cuantitativos.

\section{Resultados}

\subsection{Percepción del riesgo}

Los potenciales riesgos derivados de la realización de la investigación pueden afectar a la fiabilidad y la validez de la misma, amén de suponer una contingencia a evitar tanto por lo aconsejado en los protocolos internacionales de las asociaciones científicas, como por propia lógica al implicar a personas que trabajan dentro de la cobertura de unos centros universitarios. Para comprobar cuál es la percepción del riesgo que tienen los estudiantes sobre sus investigaciones, se les preguntó de manera expresa si creían que éstas representaban algún tipo de riesgo. Para un $68.4 \%$ de los estudiantes la respuesta fue negativa, mientras que el 31.6\% estimó que sí existían riesgos. Destaca la diferencia en la percepción del riesgo entre mujeres y hombres ya que, mientras el $75 \%$ de las mujeres entendieron que el tema carecía de riesgo, este dato baja al 54.2\% en el caso de los hombres. 
En el trabajo de campo, sin embargo, tanto alumnos como alumnas actúan de manera similar puesto que adoptan medidas de protección de los participantes y emplean el consentimiento informado en una proporción similar; esto es, un 66.7\% de las mujeres y un 69.2\% de los hombres. Del mismo modo, un 50.9\% de las mujeres y un $48 \%$ de los hombres tomaron la precaución de hacerles firmar el consentimiento informado a los participantes. Estos datos los hemos empleado como indicadores indirectos de percepción de riesgo, ya que de no darse esta condición previa no se justificaría la utilización de medidas de protección por parte de los estudiantes ni que, por tanto, solicitaran el consentimiento informado a los participantes. Así lo confirma el análisis de datos cruzados entre los ítems "percepción de riesgo" y "firma de consentimiento informado" que permite concluir que su utilización actuaría como un neutralizador de la percepción del riesgo: de quienes no solicitaron la firma del consentimiento informado, un $60 \%$ sí consideraba que la investigación suponía riesgo, mientras que de quienes sí pidieron la firma, un 57.1\% consideraba que la investigación no representaba riesgo alguno.

Para quienes respondieron afirmativamente a la pregunta de si consideraban que la investigación podía representar riesgos, la mayoría indicaba que éste era, sobre todo, para el participante individual (37.8\%), seguido por el colectivo de participantes (28.9\%), trabajadores de la organización y el estudiante (ambos con un 13.3\%), y para la organización a la que pertenece el colectivo (6.7\%). En la Tabla 2 se recogen los tipos de riesgo según el colectivo al que afectaría. El tipo de riesgo más percibido es el de estigmatización, quizá el más general y el más obvio para investigadores noveles.

\section{Tabla 2.}

Tipos de riesgo según sujeto en riesgo

\begin{tabular}{|l|l|c|}
\hline Sujeto en riesgo & Tipo de riesgo mayoritario & $\%$ \\
\hline Participante individual & Psicológico & 66.7 \\
\hline Colectivo de participantes & Estigmatización & 75 \\
\hline Trabajadores de la organización & Legal & 50 \\
\hline Estudiante & Estigmatización & 50 \\
\hline Organización a la que pertenece el colectivo & Estigmatización & 100 \\
\hline
\end{tabular}




\subsection{Valoración del método de investigación}

Por el enfoque, duración y extensión de los TFG es comprensible que las técnicas de recopilación de información preferidas por los estudiantes fueran los cuestionarios y las entrevistas en profundidad y, en menor medida, aquellas que requieren de un mayor tiempo de elaboración y empleo de medios como son la encuesta en línea o la observación participante. El análisis de las encuestas arroja que el trabajo de campo para realizar un TFG duró de media 5.87 semanas, si bien en el periodo de entre una y cuatro semanas encontramos al $42.9 \%$ de los casos, lo que refleja que el trabajo de campo que se realiza para los TFG es limitado en su extensión temporal.

La interacción con el objeto de estudio suele ser más cercana e intensa en el caso de que el alumno utilice técnicas cualitativas tales como las entrevistas en profundidad, los grupos de discusión o la observación participante. En lo que respecta a las entrevistas en profundidad, la media de entrevistas realizadas es de 11, con una duración media de 63.28 minutos. Por su parte, la duración media para la observación participante fue de 25.27 días, siendo de una duración similar al trabajo de campo con entrevistas.

Pero la elección del método no es únicamente una cuestión técnica sino que arrostra una mayor o menor interacción con participantes y colectivos vulnerables, algo que no es un tema baladí cuando trabajamos en temas sensibles. No obstante sólo para un 17.9\% el método empleado fue estresante, intrusivo para un $14.1 \%$ y arriesgado para un $6.4 \%$, lo que arroja que para el 69.2\% ( $\mathrm{N}=78)$ el método de recopilación no representara problema alguno, indicando, además, en un $72.4 \%$ de las ocasiones, que "fue el único posible" lo que muestra una visión poco crítica (o quizá muy reflexionada) sobre las técnicas de recopilación empleadas.

Un 34.4\% de los estudiantes reconoce que el participante fue quién eligió el lugar de la entrevista. De aquellos que llevaron a cabo las entrevistas "en el lugar que venía establecido por el tema", el 75\% era -por obvias razones-en prisiones. Uno de los problemas que pueden derivarse del lugar en el que se realiza el trabajo de campo es que influya en la sinceridad del entrevistado. Así, para un $64.5 \%$ el lugar sí influyó en su sinceridad. Al analizar los lugares, en relación a la "influencia que éste tiene en la sinceridad", se obtiene

\section{Revista Española de Investigación Criminológica}


que realizar las entrevistas en una institución disminuye en opinión del estudiante la sinceridad del entrevistado mientras aumenta en entornos de confianza, tales como el domicilio de los participantes.

\section{Tabla 3.}

Lugar de realización de las entrevistas

\begin{tabular}{|l|c|}
\hline Lugar de realización de las entrevistas & \% \\
\hline Domicilio de los participantes & 28.1 \\
\hline Centro penitenciario & 25 \\
\hline Cafetería & 12.5 \\
\hline ONG & 12.5 \\
\hline Lugar de trabajo participantes & 9.4 \\
\hline Centro escolar & 6.3 \\
\hline Juzgados & 6.3 \\
\hline Total & $\mathbf{1 0 0}$ \\
\hline
\end{tabular}

\subsection{Empleo de medidas de seguridad}

Cuando se habla de temas sensibles es posible que el participante no se sienta cómodo si están grabando sus respuestas ya que pueden desvelarse actuaciones ilegales o que se consideren desviadas de la norma social o, simplemente, vivencias demasiado íntimas como para dejar constancia de ellas. No obstante, sólo un 16\% reconoce que apagó la grabadora en algún momento a petición del participante. Las razones por las que lo hicieron son las habituales en los estudios con temas sensibles: el participante consideró que estaba dando mucha información, la información criticaba a los superiores, y la información revelaba acciones delictivas o asuntos reservados. Pese a que en la muestra analizada un $75 \%$ de los estudiantes grabó las entrevistas y pidió permiso para hacerlo, el $47.8 \%$ de los estudiantes cree que los participantes eran más sinceros cuando no se les grababa.

Otro de los objetivos de esta investigación era conocer si los estudiantes utilizaban medidas de seguridad para proteger a los participantes, lo que indicaría el nivel de concienciación sobre los riesgos que estos pueden correr. El análisis arroja que un $67.5 \%$ sí las utilizó frente a un $32.5 \%$ que no; pero más significativo es el dato de que el $89 \%$ de los encuestados consideró que había tomado todas las medidas de las que disponían. Las medidas 
que reconocen emplear son las habituales en los estudios etnográficos o sociológicos y giran en torno a las dos más obvias tales como no recopilar e inutilizar cierta información en su misma recopilación en el campo (Tabla 4). La falta de percepción de riesgo anticipa la probabilidad de un menor empleo de medidas de protección. Así, entre quienes consideraban que la investigación carecía de riesgo alguno, un 38.9\% no empleó medida alguna de protección, mientras que para aquellos que sí percibían riesgo sólo el 12\% no las empleó.

\section{Tabla 4.}

Medidas de protección para los participantes

\begin{tabular}{|l|c|}
\hline Medidas de protección para los participantes & \% \\
\hline Grabé o tomé notas sin indicar el nombre de las personas & 34.4 \\
\hline Ninguna en especial & 28.1 \\
\hline Usé pseudónimos & 19.8 \\
\hline No se recogían los nombres de los participantes & 11.5 \\
\hline Me vestía parecido a ellos/as para no llamar la atención & 3.1 \\
\hline Las grabaciones las guardaba al llegar a casa & 1 \\
\hline No incluir cierta información & 1 \\
\hline No identificar a las empresas & 1 \\
\hline Total & $\mathbf{1 0 0}$ \\
\hline
\end{tabular}

El 36.1\% experimentó algún tipo de problema durante la investigación y que tuvieron como fuente a la institución (36.7\%), los participantes (33.3\%), los trabajadores (13.3\%) y otros miembros del colectivo (10\%). Sin embargo, sólo el 33.3\% de ellos mencionó en su TFG la existencia de estos problemas. Por último, el 76.1\% no empleó ninguna medida de seguridad personal. Aunque las razones para esta afirmación no fueron abordadas en el cuestionario, estas podrían ser dos: o bien los estudiantes no consideran que deban autoprotegerse, o bien no conocen qué medidas de autoprotección pueden -y, en algunos casos, deben- utilizar.

Como fase final, la salida del campo es un momento importante en el que hay que cumplir con muchas de las promesas que se hicieron al ingresar y evitar dejar heridas abiertas que impidan que ulteriores investigadores -otros estudiantes de Grado-puedan trabajar allí. La autocensura - como método para evitar perjudicar a los participantes, al colectivo o a la institución- fue admitida por el $22.9 \%$ del total de los estudiantes y era más común entre 
aquellos que habían pertenecido al grupo o colectivo que investigaba: el $62.5 \%$ se autocensuró frente al $18.7 \%$ de quienes no eran miembro del grupo. Este dato indica que el haber sido miembro del grupo que se investiga puede afectar a la fiabilidad y a la validez de las investigaciones. Las razones aducidas para esta autocensura fueron: proteger la identidad de los participantes (47.4\%), información no necesaria (26.3\%), proteger mi seguridad y a los participantes $(10.5 \%)$ y me prohibieron incluirla $(10.5 \%)$.

En sentido opuesto a la autocensura está la entrega de información de manera deliberada. El gatekeeper, esto es, la persona que dio o permitió acceso al campo puede, en algún momento, solicitar al estudiante información en consideración o como contraprestación explícita por su colaboración. Así, un 32.5\% de los estudiantes reconoce que se les solicitó información durante o una vez terminada la investigación. De estos, sólo un $14.3 \%$ sostiene que no la facilitó mientras que un $39.3 \%$ lo hizo en su totalidad y, parcialmente, un 46.4\%. En el 63.6\% de los casos en que facilitaron toda la información el método de recopilación había sido la encuesta. Esto puede deberse a que este instrumento genera en los investigadores noveles una mayor sensación de distancia con el participante, quedando diluida la conciencia de sensibilidad.

\subsection{Relación con el objeto de investigación}

El investigador no es un ente neutro: su presencia en el campo afecta al rumbo de la investigación que dependerá, en gran medida, de sus características personales y su capacidad de adaptarse al objeto de estudio. Los datos muestran que el sexo y la edad afectaron de alguna manera a la investigación de los estudiantes encuestados (Tabla 5). Sin embargo, mientras la edad perjudica a los estudiantes más mayores (29-32 años) y facilita a los más jóvenes (21-23 años) -quizás debido a que la juventud inspira mayor confianza en los participantes-, el sexo favoreció o perjudicó a hombres y mujeres por igual. Esto permite concluir que la variable "sexo", no es, per se la que determinará si le perjudica o no, sino que dependerá de su interacción con el objeto de estudio. 


\section{Tabla 5.}

Efecto de la edad y sexo del estudiante en la investigación

\begin{tabular}{|c|c|c|c|}
\hline \multicolumn{2}{|c|}{ Favorece } & \multicolumn{2}{c|}{ Perjudica } \\
\hline Edad & Sexo & Edad & Sexo \\
\hline $39.2 \%$ & $33.8 \%$ & $16.2 \%$ & $14.9 \%$ \\
\hline
\end{tabular}

Estas características de sexo y edad no son modificables por los estudiantes por lo que es necesario enseñarles a gestionarlas tanto cuando les favorece como cuando les perjudica. Sin embargo, otras características como la forma de vestir o el lenguaje sí las puede alterar el estudiante. En nuestra investigación, el 54.2\% reconoce que tuvo que modificar su lenguaje y el $20.5 \%$ su forma de vestir al llegar al campo. Cruzado por "tema del TFG", se obtiene que cuando más necesarias son las adaptaciones del lenguaje y forma de vestir es cuando los estudiantes trabajan con el mundo de la prostitución (20\%). Se concluye así la importancia de la preparación previa en el aula sobre el trabajo en mundos completamente distintos a la realidad habitual del alumno.

Dadas las características de los colectivos, es de esperar que los estudiantes tengan alguna idea preconcebida antes de comenzar su trabajo. Un 56.6\% reconoce que sí las tenían, pero al cruzarlas con la variable "tema del TFG" no se obtienen conclusiones claras. Aunque esto sea así, es indudable su influencia ya que el 30.9\% reconoce de manera expresa que estas ideas preconcebidas sí le influyeron de alguna manera en su investigación.

El $18.9 \%$ cree que en algún momento los participantes pensaron que los estudiantes estaban ocultando su verdadera identidad. El análisis muestra que, cuando la institución es quien introduce al estudiante en el campo, se duda en mayor medida de sus intenciones que cuando es el tutor o un profesor quien lo hace. Informantes de la institución, personal de prisiones, policías o periodistas eran los roles con los cuales los participantes más confundían a los estudiantes. Las dudas se concentraban en torno a aquellos temas en los cuales los participantes corren mayores riesgos al tomar parte en la investigación; es decir, prisiones (42.9\%), marginación social/urbana (28.6\%) y prostitución (14.3\%), que aglutinan el 85.8\% de todos los casos. 
El humor es un recurso de primer orden en los temas sensibles. El 76.9\% de los hombres y el $68.4 \%$ de las mujeres lo utilizó en algún momento. Este uso ha sido muy positivo ya que ningún participante indica que el humor le perjudicara y un $87.1 \%$ sostiene que le favoreció; en este caso, detectamos cierta variación por sexo: mientras al $95 \%$ de los hombres les facilitó el trabajo, la cifra baja al $83.3 \%$ en el caso de las mujeres.

\subsection{Emociones y sentimientos en la investigación}

Si bien los protocolos de investigación y de seguridad pueden identificar y paliar la mayoría de los conflictos y riesgos, el estudiante se enfrentará a una serie de situaciones en una forma que dependerá en gran medida de su personalidad, grado de madurez y sensibilidad. Las situaciones vividas por los estudiantes y las emociones experimentadas en el trabajo de campo subrayan la importancia de la preparación emocional de los estudiantes antes de abordar el campo. Destaca el cómo, para un 29.2\% de los participantes, las situaciones fueron emotivas y para un $20.8 \%$ de estrés emocional, aspectos estos que nos indican la relevancia de reflexionar sobre la preparación emocional de los estudiantes antes de salir al campo.

\section{Tabla 6.}

Situaciones que vivió en el trabajo de campo

\begin{tabular}{|l|c|}
\hline Situaciones vividas & $\mathbf{\%}$ \\
\hline Ninguna & 33 \\
\hline Emotivas & 29.2 \\
\hline Estrés emocional & 20.8 \\
\hline Fraternales & 6.6 \\
\hline Incómodas & 2.8 \\
\hline Sensibles & 2.8 \\
\hline Violentas & 2.8 \\
\hline Inseguridad & 1.9 \\
\hline Total & $\mathbf{1 0 0}$ \\
\hline
\end{tabular}

De los sentimientos que estas situaciones generan en el estudiante durante el trabajo de campo predominan la frustración (12.7\%), la compasión (11.5\%) y la pena (11.5\%). Los estudiantes podían indicar hasta tres sentimientos que habían experimentado por lo que los porcentajes están en relación al total de respuestas (Tabla 7). 


\section{Tabla 7.}

Emociones y sentimientos durante el trabajo de campo

\begin{tabular}{|l|l|}
\hline Emociones y sentimientos durante el trabajo de campo & \% \\
\hline Frustración & 12.7 \\
\hline Compasión & 11.5 \\
\hline Pena & 11.5 \\
\hline Ternura & 10.3 \\
\hline Ninguna & 6.7 \\
\hline Inquietud & 6.1 \\
\hline Enfado & 5.5 \\
\hline Inseguridad & 5.5 \\
\hline Satisfacción & 4.8 \\
\hline Apego & 4.2 \\
\hline Angustia & 4.2 \\
\hline Confusión & 4.2 \\
\hline Alegría & 3.6 \\
\hline Miedo & 1.8 \\
\hline Empatía & 1.8 \\
\hline Impotencia & 1.8 \\
\hline Vergüenza & 1.2 \\
\hline Rechazo & 1.2 \\
\hline Apatía & 0.6 \\
\hline Simpatía & 0.6 \\
\hline Total & $\mathbf{1 0 0}$ \\
\hline
\end{tabular}

No obstante, de mayor interés es el cruce de estos sentimientos por la variable "sexo", donde se observa cómo el primer valor en las mujeres -la compasión, con un 47.1\%- no aparece sino hasta el noveno lugar en el caso de los hombres. Así, mientras que el sentimiento más experimentado por las mujeres es la compasión, seguido de la pena y la frustración, en el caso de los hombres predomina la frustración, seguido de la pena y la ternura.

\section{Tabla 8.}

Orden de aparición de sentimientos por sexo

\begin{tabular}{|c|c|c|c|c|}
\hline & \multicolumn{2}{|c|}{ Hombres } & \multicolumn{2}{c|}{ Mujeres } \\
\hline Orden de aparición & Emoción & $\%$ & Emoción & $\%$ \\
\hline Primero & Frustración & 47.1 & Compasión & 47.1 \\
\hline Segundo & Pena & 29.4 & Pena & 41.2 \\
\hline Tercero & Ternura & 29.4 & Frustración & 38.2 \\
\hline
\end{tabular}


Destaca que el $87.5 \%$ de los estudiantes que no experimentaron ningún sentimiento entendían que su investigación no representaba riesgo alguno. Al cruzarlo por "técnica de obtención" se observa cómo, de estos, un 71.4\%, había empleado como método la encuesta, por lo que su contacto con las experiencias y emociones de los individuos y grupos fue más exigua y distante. A aquellos que sí experimentaron sentimientos se les preguntó si las habían comentado con alguien (Tabla 9). La agregación de tutor y compañero acumulan el $41.9 \%$ de las respuestas, lo que refleja que el aula es un importante espacio donde aprender a gestionar esas emociones y sentimientos. Esto cobra más importancia si consideramos que sólo un $15.7 \%$ de los TFG se realizaron en equipo, lo que significa que el alumno se enfrenta en solitario al campo y a sus emociones.

\section{Tabla 9.}

Persona/s con quien compartió sentimientos y emociones

\begin{tabular}{|l|c|}
\hline Persona/s con quien compartió sentimientos y emociones & \% \\
\hline Amigo/a & 25.9 \\
\hline Tutor/a & 25.9 \\
\hline Compañero/a & 17 \\
\hline Pareja & 14.3 \\
\hline No las comenté con nadie & 8.9 \\
\hline Familiar & 6.3 \\
\hline Psicólogo/a & 1.8 \\
\hline Total & $\mathbf{1 0 0}$ \\
\hline
\end{tabular}

Al dejar el campo, un 53\% de los estudiantes sostiene que no experimentó sentimiento alguno (Tabla 10). De ellos, la mayoría habían utilizado como técnica de investigación la encuesta que, como se comentó más arriba, favorecía una mayor distancia entre el estudiante y su objeto de estudio, por lo que es coherente que al dejar el campo experimentara menos sentimientos. También nos encontramos con estudiantes que realizaron entrevistas en profundidad u observación participante y, aun así, no experimentaron ningún sentimiento al dejar el campo. No obstante, de aquellos que sí experimentaron algún sentimiento, predominan quienes sintieron pena (16.9\%) y liberación (10.8\%). En el primer caso, es uno de los sentimientos más básicos al enfrentarse a colectivos vulnerables. En el segundo, 
sienten liberación al dejar una realidad que no es la suya y que, en muchos casos, es angustiosa, tales como la vida en prisión o la de las prostitutas.

\section{Tabla 10.}

Sentimientos al dejar el campo

\begin{tabular}{|l|c|}
\hline Sentimientos al dejar el campo & $\mathbf{\%}$ \\
\hline Ninguno & 53 \\
\hline Pena & 16.9 \\
\hline Liberación & 10.8 \\
\hline Satisfacción & 7.2 \\
\hline Remordimiento & 3.6 \\
\hline Agradecimiento & 2.4 \\
\hline Empatía & 1.2 \\
\hline Frustración & 1.2 \\
\hline Inquietud & 1.2 \\
\hline Necesidad de continuar para hacer "algo más" & 1.2 \\
\hline Preocupación & 1.2 \\
\hline Total & $\mathbf{1 0 0}$ \\
\hline
\end{tabular}

Sólo siete (8.4\%) participantes mostraron sus dudas acerca de si sería posible volver a trabajar en el tema. Para dos de ellos la institución salió perjudicada, para otros dos su introductor ya no estaría receptivo y, para los tres restantes, quienes no estarían receptivos serían los participantes. En cualquier caso, no parece que estén alertados sobre la posibilidad de que hubieran "quemado" el campo durante su trabajo. Es cierto que la poca profundidad a la que pueden llegar por el tiempo y el conocimiento del que disponen hace que, a menos que sea por circunstancias extraordinarias, individuos, colectivo u organización puedan sin el mayor esfuerzo protegerse de un investigador junior. No obstante, este $8.4 \%$ de quienes dudan de poder volver a trabajar en el tema, tampoco es una cifra baja.

\section{Discusión y conclusiones}

Este trabajo tenía como objetivo analizar las experiencias de los estudiantes universitarios del Grado en Criminología en la elaboración de sus TFG sobre temas sensibles. Las investigaciones en estos temas, como se analizó en el marco teórico, a menudo se producen

\section{Revista Española de Investigación Criminológica}


sobre colectivos vulnerables por lo que el potencial lesivo de realizar una investigación sobre ellos es alto. La Criminología, como ciencia empírica e interdisciplinaria que estudia el delito, el delincuente, la víctima y el control social debe hacer frente en numerosas ocasiones a este tipo de investigaciones, que necesitan de unas consideraciones y aplicaciones metodológicas adaptadas a las características de su objeto y/o tema de estudio.

Primera. Los resultados parecen indicar que los estudiantes se apresuran a iniciar sus TFG sin demasiados conocimientos, reflexión metodológica y formación sobre buenas prácticas en la investigación con temas sensibles. Así, el análisis de la variable "percepción de riesgo" evidencia que la mayoría de ellos no percibió que hubiera riesgos en la investigación, en especial, las mujeres. Ello puede explicarse parcialmente por el hecho de que algunos de ellos utilizaron medidas de protección que pueden actuar como un neutralizador de la percepción del riesgo, como la firma del consentimiento informado a los participantes. No obstante, pese a que la relación estadística es significativa, ésta no es concluyente. Sí que parece coherente con el análisis del marco teórico que la estimación de probabilidad de riesgos por parte del estudiante se centre en los participantes (ya sea de forma individual o colectiva). En futuras investigaciones se comprobará si esta percepción se debe a un análisis objetivo de la realidad o bien a que el estudiante no es consciente de otros posibles riesgos de la investigación por falta de formación.

Segunda. La ausencia de reflexión y preparación que se alegaba coincide con el hecho de que los estudiantes creen que el método de investigación utilizado era el único posible, a pesar de que muchos consideran que el lugar y el modo como desarrollaron el trabajo de campo afectó a la sinceridad de las respuestas de los participantes. Además, se confirma que la mayoría utiliza consentimiento informado, grabando siempre con el conocimiento de los participantes, y toma al menos una medida para proteger su identidad. Sin embargo, pocos consideraban que podrían haber utilizado más medidas de protección a pesar de que éstas no eran abundantes.

Tercera. La variable "relación con el grupo" permite concluir que la reflexión previa al trabajo de campo sobre el rol que desempeñará el estudiante es esencial, ya que de ello dependerá la actitud de los participantes hacia éste. Ello se desprende del análisis de la influencia de variables como el sexo, la edad, la vestimenta o el lenguaje en la investigación.

Revista Española de Investigación Criminológica 
Aunque el sexo y la edad no son variables que puedan ser manipuladas a efectos de esta investigación, sí es una opción el analizar los roles disponibles para cada estudiante y escoger aquél en el que dichas variables favorezcan la investigación en lugar de perjudicarla. Ello no sólo podrá disminuir el impacto negativo de las características del estudiante en la investigación sino también reducir el número de ocasiones en las que la identidad del estudiante es confundida con la de un policía, un informante, u otro rol percibido como amenazante por los participantes.

Cuarta. Los resultados de la variable "emociones y sentimientos en la investigación" abren, en opinión de los autores, un campo nuevo de reflexión científica y pedagógica al posibilitar la conclusión de que el desarrollo de los TFG no es sólo un mero trámite académico sino que, cuando implica trabajo de campo en temas sensibles, a menudo, viene acompañado de situaciones que generan emociones y sentimientos en el estudiante para los que no ha sido preparado.

Quinta. El análisis de los resultados abre para los autores dos líneas de trabajo. En primer lugar, surge la hipótesis de que puedan existir tres perfiles de estudiantesinvestigadores. La ulterior investigación se centraría en realizar entrevistas en profundidad con algunos de los participantes de la encuesta para determinar si efectivamente se puede afirmar la existencia de una tipología y, en caso afirmativo, cuál sería la variable independiente que diferencia entre sí los distintos perfiles. Si bien los nombres definitivos de dicha tipología y sus características serían establecidos en posteriores investigaciones, indiciariamente los datos nos configuran la siguiente tipología:

Tipo I. Conformado por aquellos investigadores cuyo contacto con el objeto de estudio viene mediatizado y controlado por la institución u organización que le da acceso. De esta forma se pretende neutralizar la amenaza que representa el investigador al cual, por su posición, le son visibles realidades que no lo son para toda la organización y que hará visibles al resto a través del informe. Las organizaciones altamente controladas (Spencer, 1973) o aquellas en las que el poder es susceptible de ser usado de forma corrupta o ilegítima (Braithwaite, 1984; Punch, 1989) condicionan el cómo se realiza el estudio suponiendo una menor discrecionalidad para el investigador y una menor implicación emocional. 
Los datos indiciarios apuntarían a que este tipo I estaría muy concienciado de los riesgos de la investigación pero tendrían una mayor distancia afectiva con respecto al objeto de estudio.

Tipo II. Compuesto por estudiantes atraídos por la oportunidad de conocer de cerca la realidad que habían estudiado de forma teórica durante cuatro años y seducidos por la posibilidad de comprender a un "otro exótico" del que hablaban Denzin y Lincoln (2006). Exótico, innovador y desafiante, serían los componentes que Nordstrom (1994) resume en “diversión”, el elemento sin el cuál para él no sería fácil involucrarse en el campo de los temas sensibles. Por esto, este tipo de estudiantes estaría caracterizado por desarrollar una relación más cercana con su objeto de estudio y, en consecuencia, experimentar en mayor número e intensidad emociones y sentimientos en el trabajo de campo.

Tipo III. Motivado por la inexistencia de comités de ética que revisen estas investigaciones y la ausencia de sensibilidad y conocimiento metodológico sobre cómo abordar estos temas, es esperable la detección de un grupo poco consciente de la peculiaridad de estas investigaciones y que se acercaría a ella con los mismos bártulos que a cualquier otro tema. Como sostenía Humphreys (1970), un perfil que quizá se anima por un cierto espíritu aventurero pero muy desubicado en el trabajo de campo. Serían así aquellos estudiantes que no son conscientes de los riesgos ni se involucran con su objeto de estudio, por lo que tampoco experimentarían emociones ni sentimientos durante el trabajo de campo o a la salida del mismo.

Sexta. Junto a la potencial tipología, la segunda línea de trabajo que se abre es referente a los resultados obtenidos sobre los sentimientos y emociones experimentados por los estudiantes y que hacen necesario medir la gestión de las emociones en el aula y en las investigaciones universitarias. Esto podría realizarse mediante la estandarización de un cuestionario que se desarrollaría partiendo de instrumentos ya testados como el de Fredrickson (2009) sobre emociones positivas y negativas. El objetivo de esta segunda línea de investigación sería adaptar estos instrumentos al contexto de la educación emocional en las aulas.

Séptima. A lo largo de toda la investigación hemos comprobado el papel central que desarrollan los profesores y los tutores de TFG. Por esto, proponemos que aquellos Revista Española de Investigación Criminológica 
estudiantes que elaboren sus TFG sobre temas sensibles puedan participar en un seminario antes de entrar en el campo. Esto le permitiría confrontar el yo como persona y como investigador con las personas, situaciones y fenómenos con los cuales se va a encontrar, como sugería Woodthorpe (2011). Estas pequeñas reuniones se convertirían, en palabras de Warren et al. (2003), en esas ventanas para analizar cómo los investigadores gestionan su estrés y su tristeza. Como decía Kimmel (1988), cuando estos juicios éticos se nos plantean en medio de la investigación es ya, sencillamente, demasiado tarde para darles una solución adecuada. Por eso, este entrenamiento les prepararía para hacer frente a diversos dilemas morales y éticos durante la elaboración del trabajo y a los cuáles los comités de ética difícilmente pueden adelantarse. En base a los descubrimientos de esta investigación, los autores (DíazFernández, 2018; Díaz-Fernández \& del Real Castrillo, 2017) elaboraron un taller para guiar a docentes y estudiantes a prepararse y reflexionar sobre cómo adentrarse y trabajar en estos temas sensibles.

En definitiva, en opinión de los autores, es necesario introducir de forma paulatina a los estudiantes de Criminología en aquellos elementos básicos de la investigación en temas sensibles en la confianza de que les facilitará su trabajo a lo largo de su proceso formativo. Quizá por esto Hamm y Ferrel (1998) invitaban a los residentes del Hotel Criminología a abandonarlo por un momento e ir a las calles, a salas de estar, a organizaciones, a clubs juveniles... Invitan a situarse tan cerca cómo se pueda de los autores de un crimen, de los desviados, de las víctimas de los agentes del orden, a ubicarse de la mejor forma que se pueda y por el mayor período de tiempo posible dentro de sus vidas, dentro de sus experiencias vivas de desviación y de crimen. No será una experiencia agradable y si el peligro y el dolor se hacen intensos - concluyen- "felicítate por ello, ya que es lo más cerca del camino que te encontrarás dentro de la humanidad del crimen y de la desviación”. 


\section{Referencias}

Barnes, J. A. (1979). Who Should Know What? Social Science, Privacy and Ethics. Harmondsworth: Penguin.

Braithwaite, J. (1984). Corporate Crime in the Pharmaceutical Industry. Londres: Routledge \& Kegan Paul.

Brewer, J. (1993). Sensitivity as a Problem in Field Research. En C. Renzetti \& R. Lee (eds.), Researching Sensitive Topics (pp.125-145). London: Sage.

Bryman, A. (1988). Quantitative and Quality in Social Research. Londres: Unwin Hyman.

Denzin, N. R., \& Lincoln, Y. S. (2006). Handbook of Qualitative Research (3 ${ }^{\mathrm{a}}$ ed.). Thousand Oaks: Sage.

Díaz-Fernández, A. M. (2018). La investigación en temas sensibles en Criminología y Seguridad. Madrid: Ed. Tecnos.

Díaz-Fernández, A. M., \& del Real Castrillo, C. (2017). Cómo tutorizar TFG y TFM en temas sensibles, Actas de la II Jornada de Innovación Docente (pp.451-456). Universidad de Cádiz.

Dickson-Swift, V., James, E. L., Kippen, S., \& Liamputtong, P. (2009). Researching Sensitive Topics: Qualitative Research as Emotion Work. Qualitative Research, 9(1), 6179. DOI: $10.1177 / 1468794108098031$

Dickson-Swift, V., James, E. L., Kippen, S., \& Liamputtong, P. (2007). Doing sensitive research: what challenges do qualitative researchers face? Qualitative Research, 7(3), 327-353. DOI: $10.1177 / 1468794107078515$

Flaskerud, J. H., \& Winslow, B. J. (1998). Conceptualising Vulnerable Populations in Health-Related Research. Nursing Research, 47(2), 69-78. DOI: 10.1097/00006199199803000-00005

Fredrickson, B. (2009). Positivity. New York: Crown Publishers.

Hamm, M., \& Ferrell, J. (1998). Confessions of Danger and Humanity. En J. Ferrell \& M. Hamm (eds.), Ethnography at the Edge (pp.254-273). Boston, MA: Northern University Press.

Howell, N. (1990). Surviving fieldwork: A report of the advisory panel on health and safety in fieldwork. Washington DC: American Anthropological Association.

Humphreys, L. (1970). Tearoom Trade: A study of Homosexual Encounters in Public Places. Londres: Gerald Duckworth.

Kimmel, A. (1988). Ethics and Values in Applied Social Research. Londres: Sage. DOI: 10.4135/9781412984096

Kleinman, S. (1991). Field-workers' feelings: What we feel, who we are, how we analyze. En W. Shaffir \& R. A. Stebbins (eds.), Experiencing fieldwork: An inside view of qualitative research (pp.184-195). Newbury Park, CA: Sage. DOI: 10.4135/9781483325514.n15

Low, G., \& Organista, K. C. (2000). Latinas and sexual assault: Towards culturally sensitive assessment and intervention. Journal of Multicultural Social Work, 8(1-2), 131-157. DOI: 10.1300/J285v08n01_06

Lowe, P. (2015). Lessening sensitivity: student experiences of teaching and learning sensitive issues. Teaching in Higher Education, 20(1), 119-129. DOI: 10.1080/13562517.2014.957272

Revista Española de Investigación Criminológica

Artículo 2, Número 16 (2018)

www.criminologia.net

ISSN: 1696-9219 
Macklin, R. (1980). The Problems of Adequate Disclosure in Social Science Research. En T. L. Beauchamp, R. R. Faden, R. J. Wallace, L. Walter (eds.), Ethical Issues in Social Science Research (pp.193-214). Baltimore, Maryland: The Johns Hopkins University.

Mead, M. (1972). Blackberry Winter, My Earliest Years. Nueva York: William Morrow.

Moore, L. W., \& Miller, M. (1999). Initiating Research with Doubly Vulnerable Populations. Journal of Advanced Nursing, 30(5), 1034-1040. DOI: 10.1046/j.13652648.1999.01205.x

Mosher, D. L. (1988). Balancing the Rights of Subjects, Scientists, and Society: 10 Principles for Human Subject Committees. The Journal of Sex Research, 24, 378-385. DOI: $10.1080 / 00224498809551438$

Nilan, P. (2002). Dangerous fieldwork' re-examined: the question of researcher subject position. Qualitative Research, 2(3), 363-386. DOI: 10.1177/146879410200200305

Nordstrom, C. (1994). War on the Front Line. En Nordstrom, C. \& Robbe, A C. G. M. Fieldwork under fire. Contemporary Studies of Violence and Survival. Berkeley: University of California Press.

Nyamathi, A. (1998). Vulnerable Populations: A continuing Nursing Focus. Nursing Research, 47(2), 65-66. DOI: 10.1097/00006199-199803000-00001

O'Byrne, D. (2014). The Places and Spaces of Human Rights Education. Enhancing Learning in the Social Sciences, 6(1), 66-74. DOI: 10.11120/elss.2014.00021

Punch, M. (1989). Researching police deviance: a personal encounter with the limitations and liabilities of fieldwork. British Journal of Sociology, 40(2), 177-204. DOI: $10.2307 / 590268$

Real Decreto 1393/2007, de 29 de octubre, por el que se establece la ordenación de las enseñanzas universitarias oficiales.

Renzetti, C. M., \& Lee, R. (1993). Researching Sensitive Topics. Londres: Sage.

Sieber, J. E., \& Stanley, B. (1988). Ethical and professional dimensions of socially sensitive research. American Psychologist, 43(1), 49-55. DOI: 10.1037/0003-066X.43.1.49

Silva, M. C. (1995). Ethical Guidelines in the Conduct, Dissemination, and Implementation of Nursing Research. Washington, DC: American Nurses Publishing.

Spencer, G. (1973). Methodological issues in the study of bureaucratic élites: a case study of West Point. Social Problems, 21(1), 90-103. DOI: 10.2307/799979

Warren, C. A., Barnes-Brus, T., Burgess, H., Wiebold-Lippisch, L., Hackney, J., Harkness. G., ... R. Shuy (2003). After the Interview. Qualitative Sociology, 26(1), 93-110. DOI: 10.1023/A:1021408121258

Wolf, L. E. (2009). IRB Policies regarding Finder's Fees and Role Conflicts in Recruiting Research Participants. IRB: Ethics and Human Research, 31(1), 14-19.

Woodthorpe, K. (2011). Researching death: methodological reflections on the management of critical distance. International Journal of Social Research Methodology, 14(2), 99-109. DOI: $10.1080 / 13645579.2010 .496576$

\section{Agradecimientos}

Queremos agradecer a Elena Trabajo sus comentarios al cuestionario implementado y su colaboración en su difusión. Así mismo, los comentarios realizados a los resultados

Revista Española de Investigación Criminológica

26

Artículo 2, Número 16 (2018)

www.criminologia.net

ISSN: 1696-9219 
provisionales dentro del proyecto de investigación "Valoración de la eficiencia e impacto de la respuesta social y jurídica a la pederastia a partir del análisis criminológico" DER201567303-P y dirigido por la Dra. Rodríguez Mesa.

Antonio M. Díaz Fernández es Doctor en Ciencia Política por la Universidad de Barcelona, y profesor Titular de Derecho Penal en la Universidad de Cádiz. Ha coordinado el Máster Oficial en Sistema Penal y Criminalidad y es vice editor de The International Journal of Intelligence, Security, and Public Affairs. Entre sus áreas de interés se encuentran los servicios de inteligencia y los métodos de investigación en temas sensibles.

(iD) https://orcid.org/0000-0002-2376-0374

Cristina del Real Castrillo es Graduada en Criminología por la Universidad de Sevilla y ha realizado el Máster Oficial en Sistema Penal y Criminalidad en la Universidad de Cádiz. En ambas titulaciones obtuvo el premio extraordinario fin de estudios de su promoción. Actualmente es investigadora predoctoral en la Universidad de Cádiz, donde realiza su Tesis doctoral sobre seguridad en las ciudades inteligentes.

iD https://orcid.org/0000-0003-3069-4974 